\title{
Resituer l'adoption des propositions techniques de la recherche dans les stratégies d'adaptation des exploitants agricoles familiaux
}

\author{
P. Pédelahore ${ }^{1 *}$ R. Tchatchoua ${ }^{2}$ M. Tonka ${ }^{3}$ \\ M. Ntsama ${ }^{3}$ N. Andrieu ${ }^{1,4}$
}

\section{Mots-clés}

Exploitation agricole familiale Adoption de l'innovation - Politique de développement - Cameroun.

\begin{abstract}
Résumé
L'innovation technique est souvent présentée comme le levier principal de l'amélioration des performances économiques et des conditions de vie des exploitants agricoles familiaux (EAF). Elle constitue de ce fait un moyen d'adaptation face à la variabilité de I'environnement socio-économique. L'objectif de cette étude a été d'analyser la place du recours à l'adoption de propositions techniques issues de la recherche au sein de l'ensemble des stratégies d'adaptation mobilisées par les producteurs pour préserver, voire pour améliorer, leurs conditions de vie et celles de leurs descendants. A travers la réalisation d'entretiens semi-directifs et directifs auprès d'un échantillon représentatif d'EAF du Grand Sud Cameroun, cette étude a montré que le développement des migrations et des activités non agricoles, I'extension des surfaces cultivées, et la diversification des productions agricoles marchandes étaient des stratégies d'adaptation plus fréquemment mobilisées que l'adoption de propositions techniques. Cette étude a souligné le fait que l'amélioration des itinéraires techniques et des performances des exploitations familiales ne peuvent constituer la seule orientation des politiques de recherche et de développement. L'augmentation des mobilités spatiales et professionnelles des producteurs encourage à développer des politiques de recherche et de développement plus attentives à la gestion des territoires et des hommes et aux interactions entre les différents secteurs de l'économie nationale.
\end{abstract}

\section{INTRODUCTION}

Les trois quarts des habitants pauvres des pays en développement vivent dans les espaces ruraux et dépendent directement ou indirectement de l'agriculture pour leur subsistance (2). Dans ces pays, et en Afrique subsaharienne en particulier, la Banque mondiale (2) présente l'agriculture comme un instrument pour la réduction de la proportion de la population vivant dans l'extrême pauvreté et souffrant de faim chronique, et pouvant favoriser la croissance économique, à condition que s'opère une révolution au niveau de la productivité des exploitations agricoles familiales.

1. Cirad, UMR Innovation, 73 rue Jean-François Breton, 34398 Montpellier Cedex 5 , France.

2. Université de Dschang, Dschang, Cameroun

3. IRAD, Yaoundé, Cameroun.

4. Cirdes, Bobo-Dioulasso, Burkina Faso.

* Auteur pour la correspondance

Tél. : +33 (0)4 67615754 ; fax : +33 (0)4 67614415

E-mail : philippe.pedelahore@cirad.fr
Au Cameroun, le taux de pauvreté des ménages ruraux a augmenté de 52,1 à 55 p. 100 entre 2001 et 2007 (33). La pauvreté rurale est un phénomène ancien et récurrent. L'amélioration des revenus des exploitants agricoles, grâce à l'amélioration des rendements et de la productivité du travail, constitue depuis plusieurs décennies l'une des priorités affichées par les différents gouvernements et organismes de recherche et de développement camerounais $(16,33,34,35)$. Ainsi, même si depuis une vingtaine d'années les objectifs de compétitivité et de respect de l'environnement sont également mentionnés, la promotion de l'innovation technique constitue depuis 50 ans l'un des principaux objectifs des institutions de recherche et de développement agricole de ce pays. Cependant, force est de constater que cette modernisation peine à se réaliser. L'appareil de production agricole camerounais reste à 80 p. 100 détenu par une petite agriculture familiale qui utilise peu ou pas d'intrants, très peu mécanisée et dont les itinéraires techniques ont généralement peu évolué (32). Les rendements et la productivité du travail de cette agriculture familiale restent faibles (32). 
Face à ce constat, de nombreux travaux visent à développer des méthodes de recherche plus participatives, des inventions techniques plus adaptées aux conditions de production des petits agriculteurs et des services de conseil et de crédit agricole plus efficaces $(17,21,31)$. Ces évolutions vont sans nul doute dans le bon sens, mais la question que l'on peut aussi se poser est la suivante : l'adoption de propositions techniques issues de la recherche en vue de moderniser leur agriculture, constitue-t-elle la principale stratégie d'adaptation mobilisée par les exploitants agricoles familiaux (EAF) pour préserver, voire pour améliorer leurs conditions de vie et celles de leurs descendants?

Selon Yung et Zaslavsky (49), la stratégie des EAF est « l'art d'acteurs qui font concourir des moyens agricoles, mais non exclusivement tels, pour atteindre des objectifs de maintien, croissance et reproduction de leur unité de production familiale ». Elle est dépendante des moyens de production dont disposent les producteurs et de l'environnement pédoclimatique et socio-économique dans lequel ils exercent leurs activités (7). L'adaptation fait référence à une stratégie d'un système (famille, communauté, groupe, pays, etc.) cherchant à faire face ou à s'ajuster à un environnement nouveau ou changeant, un choc, un risque, des dommages potentiels ou à tirer profit d'opportunités $(6,22)$. Scoones (42) identifie quatre types de stratégies d'adaptation des EAF : l'intensification de la production agricole, l'extensification de la production agricole, la diversification des moyens d'existence et des activités, et la migration.

Dans le Grand Sud Cameroun, la période 1960-1987 a été marquée par l'accroissement des opportunités d'emplois urbains mieux rémunérés que l'activité agricole, et les années 1990 par une baisse drastique des cours du café et du cacao, la dévaluation du franc CFA et l'augmentation de l'écart entre le coût de la vie et le prix de vente des productions agropastorales. Les études qui ont cherché à caractériser les stratégies d'adaptation mobilisées par les acteurs face à cette variation de l'environnement socio-économique sont relativement anciennes $(1,3,13,18,23,28,29,40,48)$. Ainsi, la question du lien entre ces stratégies et l'innovation technique a été peu analysée durant ces deux dernières décennies.

Notre hypothèse a été que les EAF développent une gamme importante de stratégies d'adaptation pour préserver, voire pour améliorer, leurs conditions de vie et celles de leurs descendants, au sein desquelles l'adoption de propositions techniques est marginale. L'objectif de cet article a été d'analyser la place de l'adoption de propositions techniques issues de la recherche dans le cadre plus général de la gamme des stratégies mobilisées par les EAF.
Cette étude, menée auprès d'EAF du Grand Sud Cameroun, ne cible pas une technologie particulière mais analyse dans quelle mesure les propositions techniques visant à intensifier les systèmes de production ressortent du discours de ces EAF comme l'une des stratégies d'adaptation qu'ils mobilisent.

Après avoir présenté la méthode utilisée pour cette étude, ont été analysées la gamme des stratégies mobilisées par les EAF, puis les modalités et l'importance de la stratégie d'adaptation la plus fréquemment citée par les EAF. La discussion a porté sur la place de la technique dans les stratégies d'adaptation des EAF ainsi que sur les apports méthodologiques et opérationnels de cette étude. La conclusion a montré en quoi les enseignements de cette étude pouvaient contribuer à la réorientation des politiques de recherche et de développement agricoles.

\section{MATERIEL ET METHODES}

L'analyse privilégiée a porté sur des situations contrastées représentant une partie de la diversité du Grand Sud Cameroun. Le choix des quatre sites d'étude (Batcham, Galim, Obala et Ayos) a été basé sur quatre critères de stratification de l'espace (tableau I et figure 1) :

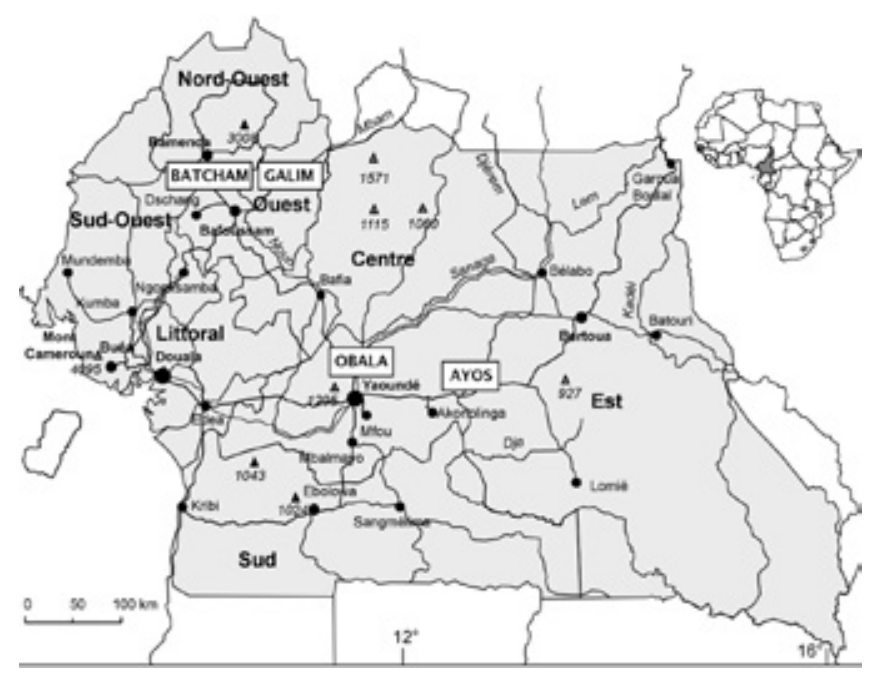

Figure 1 : localisation des quatre sites d'étude dans le Grand Sud Cameroun.

\section{Tableau I}

Caractéristiques des quatre sites d'étude

\begin{tabular}{|c|c|c|c|c|}
\hline & Batcham & Galim & Obala & Ayos \\
\hline $\begin{array}{l}\text { Densité de population } \\
\left(\mathrm{nb} \text {. habitants } / \mathrm{km}^{2}\right)\end{array}$ & $>150$ & $50<x>100$ & $100<x>150$ & $<30$ \\
\hline $\begin{array}{l}\text { Caractéristiques } \\
\text { morphopédologiques }\end{array}$ & $\begin{array}{l}\text { Hauts plateaux } \\
(>1500 \mathrm{~m}) \text { sur sols } \\
\text { volcaniques }\end{array}$ & $\begin{array}{l}\text { Hauts plateaux } \\
(>1500 \mathrm{~m}) \text { sur sols } \\
\text { volcaniques }\end{array}$ & $\begin{array}{l}\text { Plateaux (600 à } \\
800 \mathrm{~m} \text { ) sur sols } \\
\text { latéritiques }\end{array}$ & $\begin{array}{l}\text { Plateaux (600 à } \\
800 \text { m) sur sols } \\
\text { latéritiques }\end{array}$ \\
\hline Système de production & Vivriers + café & $\begin{array}{l}\text { Vivriers + maraîchage } \\
\text { (café résiduel) }\end{array}$ & $\begin{array}{l}\text { Cacao + vivrier + } \\
\text { maraîchage }\end{array}$ & Vivrier + cacao + café \\
\hline $\begin{array}{l}\text { Intégration aux réseaux } \\
\text { routiers et marchands }\end{array}$ & Bonne et ancienne & Bonne et ancienne & $\begin{array}{l}\text { Très bonne et très } \\
\text { ancienne }\end{array}$ & Moyenne et récente \\
\hline
\end{tabular}


densité de population, caractéristiques morphopédologiques, systèmes de production et intégration aux réseaux routiers et marchands. La caractérisation des stratégies d'adaptation a été réalisée par des entretiens auprès des EAF de ces sites durant trois phases successives.

La première phase visait à identifier les différentes stratégies d'adaptation mises en œuvre par les EAF. Des entretiens de type semi-directifs ont été menés auprès de 122 EAF sélectionnés au hasard et appartenant dans leur majorité à diverses organisations paysannes (OP) des sites d'étude. Les exploitants ont été interrogés sur la façon dont ils s'y prenaient pour préserver ou améliorer leurs conditions de vie et celles des membres de leur famille. Le discours de ces individus a fait l'objet d'une analyse de contenu qui est une technique de recherche pour la description objective, systématique et quantitative du contenu manifeste de la communication (4). Cette analyse des éléments du discours de la personne interrogée (11) cherche à catégoriser les différentes stratégies mentionnées par ces personnes. La fréquence de ces catégories a été calculée en fonction du nombre d'individus les ayant mentionnées par rapport au nombre total d'individus interrogés.

La deuxième phase visait à caractériser les modalités de mise en œuvre de la stratégie la plus fréquemment citée à l'issue de la première phase : la diversification des activités et des revenus. Elle a consisté en des entretiens de type directif auprès de 138 EAF sélectionnés au hasard sur les quatre sites d'étude. Comme nous étudiions la même population agricole et nous étions en contact avec les mêmes OP, certains de ces $138 \mathrm{EAF}$ ont été les mêmes que ceux retenus dans l'échantillon de la phase 1 comprenant 122 EAF. Cependant, comme ces deux tirages ont été effectués au hasard, la majorité des EAF de ces deux échantillons ont été différents. Une typologie des $138 \mathrm{EAF}$, basée sur l'importance relative des diverses sources de revenus monétaires (cultures, élevages, activités non agricoles) dont bénéficiait l'ensemble des ménages agricoles, a été réalisée. Cette typologie, dont seuls quelques uns des principaux résultats ont été présentés, a permis de constituer un échantillon de $38 \mathrm{EAF}$, représentatifs des différents types identifiés. La représentativité de cet échantillon était basée sur le fait que les EAF retenus recouvraient la totalité des types identifiés et que le nombre d'EAF retenu par type était fonction de l'importance quantitative de chaque type dans la population étudiée. L'effectif total de cet échantillon (38 EAF) a été un compromis entre la nécessité de retenir un nombre d'EAF suffisamment élevé pour que l'échantillon respecte le mieux possible l'importance quantitative de chaque type dans la population étudiée, et la prise en compte des contraintes financières qui limitaient le nombre d'enquêtes réalisables. C'est cet échantillon de $38 \mathrm{EAF}$ qui a été utilisé pour la réalisation des enquêtes conduites dans la troisième phase des recherches.

Durant cette troisième phase, les chefs de famille de ces 38 exploitations agricoles ont été interrogés, ainsi que leur parentèle (père/ mère, frères/sœurs, épouses, enfants) (figure 2).

Les entretiens semi-directifs ont permis de retracer le «parcours de vie » de trois générations durant le $\mathrm{XX}^{\mathrm{e}}$ siècle. Les parcours de vie de 576 individus ont ainsi été renseignés : les 38 chefs d'exploitation et les 538 membres de leur parentèle. Le parcours de vie étudie les multiples aspects du déroulement de la vie humaine dans son extension temporelle et dans son cadrage sociohistorique (14). Pour chacun de ces parcours de vie, le pourcentage de temps consacré à l'activité agricole a été calculé selon la méthode présentée dans la figure 3 . Cette méthode a été définie pour prendre en compte les périodes où l'exploitant menait en même temps des activités agricoles et non agricoles, et les périodes où se succédaient les activités agricoles et non agricoles.

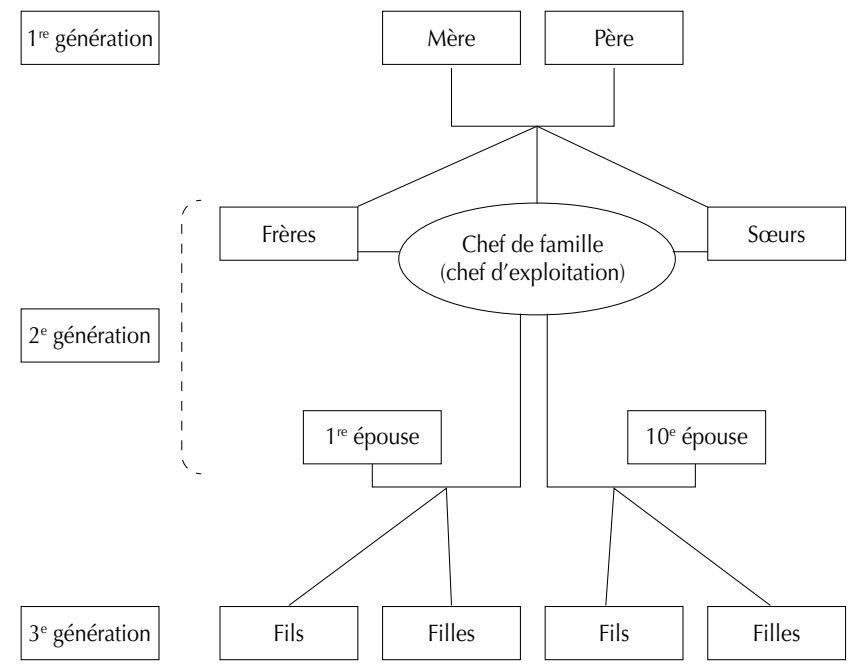

Figure 2 : individus interrogés lors de la troisième phase d'enquêtes.

Exemple de calcul pour un homme de 60 ans

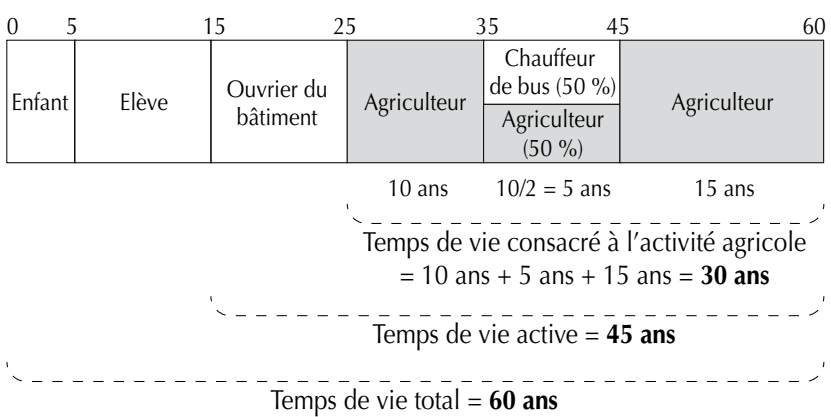

Pourcentage du temps de vie consacré à l'activité agricole = 30 ans $/ 60$ ans $=50 \%$

Pourcentage du temps de vie active consacré à l'activité agricole = 30 ans $/ 45$ ans $=66 \%$

Figure 3 : mode de calcul du temps de vie consacré à l'activité agricole.

Les 576 individus ont ensuite été classés en fonction de l'importance de l'activité agricole dans leur parcours de vie. Dans cet article n'ont été présentés en détail que les résultats concernant le site d'Obala et en particulier les données concernant les parcours de vie des 122 individus * âgés de plus de trente ans au moment de l'enquête.

\section{RESULTATS}

Les résultats obtenus sur la diversité des différentes stratégies d'adaptation des EAF ont d'abord été présentés, puis ceux concernant l'importance relative actuelle des différentes sources de revenus monétaires. Enfin, cette étude a montré comment les chefs d'exploitations agricoles et leur parentèle construisaient des parcours de vie mêlant activités agricoles et non agricoles.

* Ce nombre de 122 individus, dont les parcours de vie ont été renseignés par la troisième phase des recherches, ne doit pas être confondu avec l'échantillon de 122 EAF utilisé dans la première phase des travaux. Ce n'est qu'une coïncidence si ces deux nombres sont identiques. 


\section{Des stratégies centrées sur la diversification des productions et des activités}

L'analyse des discours recueillis lors de la première phase de l'étude a montré que les 122 EAF développaient six grands types de stratégies d'adaptation (tableau II).

La stratégie d'adaptation citée par plus de 66 p. 100 des EAF interrogés pour trois des quatre sites d'étude, et entre 33 et 66 p. 100 pour le quatrième, a été le développement d'activités non agricoles. L'agriculture est en effet considérée comme une activité « abandonnée par l'Etat», pénible physiquement, risquée et peu rémunératrice. Le statut d'agriculteur est souvent perçu, non comme un choix positif ou comme un vrai métier, mais comme une situation contrainte : « il fallait que quelqu'un de la famille garde la terre », «c'est mon père qui m'a ramené de la ville pour me marier au village », " je n'étais pas fort à l'école ». Le développement d'activités non agricoles (petit commerce, ouvriers du bâtiment ou des chantiers, routiers, chauffeurs) implique souvent la migration du chef d'exploitation vers les zones urbaines pour des périodes plus ou moins longues. Dans d'autres cas, les activités agricoles et non agricoles sont menées de front (par exemple, agriculteur et chauffeur de bus, agriculteur et commerçant). Cette stratégie de "sortie de l'agriculture » concerne également l'avenir des enfants des EAF : « je ne veux pas que mes enfants soient planteurs comme moi ! ». Les EAF consacrent une part importante de leurs revenus au paiement des frais de scolarité de leurs enfants (écoles et lycées publics ou privés). Pour les ménages peu fortunés, dont les revenus monétaires annuels sont de l'ordre de 100000 à $500000 \mathrm{FCFA} / \mathrm{an}$ (soit 150 à $750 € /$ an ; $1 € \approx 656$ FCFA), l'effort financier consenti pour l'éducation et la formation professionnelle des enfants peut fréquemment représenter jusqu'à 50 p. 100 de ces revenus. On observe ainsi que les stratégies visant à sortir de l'agriculture surdéterminent une partie des stratégies agricoles et des possibilités d'investissement dans l'outil de production ou les intrants agricoles.

La seconde stratégie d'adaptation commune à l'ensemble des quatre sites, et mentionnée par plus de 66 p. 100 des personnes

\section{Tableau II}

Stratégies des EAF * pour les sites de Batcham, Galim, Obala et Ayos

Stratégie des exploitants Batcham Galim Obala Ayos
par site

\section{Développer des activités} et des revenus non agricoles

Diversifier les productions agricoles marchandes

Migrer à la recherche de terres fertiles

Augmenter les surfaces cultivées / actif Vendre au meilleur prix Améliorer les performances des itinéraires techniques (variétés améliorées surtout)

* Exploitants agricoles familiaux

+++ Citée par plus de $66 \%$ des personnes interrogées ; ++ citée par 33 à $66 \%$ des personnes interrogées ; + citée par moins de $33 \%$ des personnes interrogées : $0=$ non citée par les personnes interrogées interrogées sur deux des quatre sites, et entre 33 et 66 p. 100 des personnes interrogées pour les deux autres, a été la diversification des productions végétales et animales marchandes. La libéralisation des filières café et cacao, l'instabilité des prix de ces produits d'exportation, ainsi que le développement de la demande urbaine en denrées alimentaires ont conduit la plupart des EAF à développer fortement les cultures vivrières et maraîchères et, pour certains d'entre eux, les élevages de volailles ou de porcs.

Les deux stratégies suivantes ont été la migration temporaire ou définitive et l'augmentation des surfaces cultivées par actif. Ces stratégies étaient étroitement liées à la densité de population du site considéré. Dans les zones comme Batcham et Obala, où la densité de population dépassait les 100 habitants $/ \mathrm{km}^{2}$, la migration temporaire ou définitive à la recherche de nouvelles terres cultivables a été citée par plus de 66 p. 100 des EAF interrogés, alors qu'elle n'a pas $(0$ p. 100) ou a été peu mentionnée (<33 p. 100) par les exploitants des zones moins peuplées d'Ayos et de Galim. Dans ces dernières, c'était l'augmentation des surfaces cultivées par actif dans leur terroir d'origine qui a permis aux exploitants de maintenir, voire d'augmenter, leurs revenus monétaires. Ainsi, par exemple, à Ayos les femmes ont commencé, dès 1990, à augmenter les surfaces de cultures vivrières devenues, surtout à partir de « l'arrivée du goudron » en 1992, des cultures marchandes. Elles ont pu, en créant des groupes féminins d'entraide, peu usités auparavant, mettre en place de vastes surfaces de culture de manioc ( $>1 \mathrm{ha}$ ) dont les productions ont été commercialisées. De même les hommes se sont mis depuis 1992, sans abandonner leurs parcelles de café et de cacao, à cultiver de grandes parcelles de bananier plantain ( $>1 \mathrm{ha}$ ) dont la vente leur a permis d'augmenter leurs revenus monétaires (« on ne va pas se laisser dépasser par les femmes quand même !»).

La stratégie visant à vendre au meilleur prix a été citée par 33 à 66 p. 100 des personnes interrogées dans trois des quatre sites et moins de 33 p. 100 dans le quatrième. La disparition des filières administrées (cacao et café) qui garantissaient un prix à la récolte, même si celui-ci pouvait varier d'une année à l'autre, a conduit les EAF à s'insérer dans les marchés concurrentiels et fluctuants des denrées vivrières ou maraîchères. Les nouvelles conditions de vente de leurs produits les ont amenés à mettre en place des stratégies leur permettant de profiter au mieux des variations de prix. Les cultures de contre-saison, le stockage sur pied du maïs ou du macabo en attendant la remontée des cours de ces denrées, ont été autant de stratégies visant à obtenir les prix les plus rémunérateurs pour les denrées produites. Les EAF ont également tenté, avec des résultats plus ou moins convaincants, de mettre en place des OP permettant à travers la vente groupée de prospecter de nouveaux marchés et de mieux négocier les prix de vente.

La stratégie visant à améliorer les performances des itinéraires techniques a été la moins citée. A part pour Galim, une forte dynamique a été observée autour de la production intensive et de la vente des produits maraîchers, moins de 33 p. 100 des EAF interrogés ont mentionné cette stratégie. Des exemples intéressants de valorisation des produits de la recherche ont été cités lors de ces entretiens. Ils concernaient surtout l'adoption de matériel végétal amélioré (maïs, pomme de terre, palmier à huile, etc.) ou de nouvelles techniques de multiplication du matériel végétal (pour le plantain, par exemple). Mais, en dehors de quelques cultures maraîchères (comme la tomate, le piment), peu de recommandations issues de la recherche ou de la vulgarisation portant sur une intensification à base d'intrants agricoles (engrais ou pesticides) ou de nouvelles pratiques techniques étaient adoptées. Les itinéraires techniques de nombreuses spéculations, en particulier vivrières (macabo, manioc, arachide, etc.), faisaient l'objet d'un nombre limité d'innovations techniques. Pour les cultures pérennes comme 
le café ou le cacao, certaines des personnes interrogées ont indiqué que l'on assistait même plutôt à une baisse du respect des recommandations techniques : diminution des doses ou disparition des applications d'engrais sur café, diminution du nombre ou disparition des traitements contre les capsides du cacaoyer. Cette régression est due aux périodes de baisse des prix de vente de ces denrées et au retrait des appuis étatiques à ces filières : suppression des subventions aux intrants et disparition des brigades phytosanitaires. De manière plus générale, et bien que les différentes enquêtes de l'étude n'aient pas comporté de questions précises et systématiques sur le montant des revenus annuels des EAF, les discours des EAF enquêtés ont montré que cette difficulté d'intensification de la production agricole à base d'intrants se heurtait aux faibles disponibilités monétaires de la grande majorité des EAF et à la priorité donnée à la résolution des problèmes de santé (maladie) ou sociaux (participations financières lors des deuils), et à l'éducation des jeunes.

\section{Des revenus non agricoles souvent importants}

Les résultats de la seconde phase de l'étude (tableau III) ont montré que si pour Galim les revenus provenaient à 90 p. 100 des activités agricoles (cultures et élevage), pour les trois autres sites (Batcham, Obala et Ayos), les activités non agricoles procuraient de l'ordre de 30 p. 100 des revenus totaux des ménages agricoles (de 25 à 34 p. 100).

Ces moyennes par site masquaient de fortes disparités entre les ménages de chacun des sites. La réalisation d'une typologie basée sur l'importance relative des différentes sources de revenus monétaires (cultures, élevages, activités non agricoles) a révélé que les activités non agricoles pouvaient procurer à certains ménages la majorité de leurs revenus. Ainsi à Batcham, les revenus non agricoles représentaient plus des trois quarts des revenus d'un ménage sur trois. A Ayos et à Obala, un ménage sur cinq tirait plus de 50 p. 100 de ses revenus des activités non agricoles.

Le tableau III montre également que la mobilité spatiale des chefs d'exploitation était non négligeable pour toutes les zones, qu'elles aient été ou non proches des centres urbains. Ces chefs d'exploitations ont passé en moyenne entre sept et onze ans en dehors de leur village, soit pour des activités liées à leur formation de base ou professionnelle, soit pour développer une activité salariale ou privée, généralement non agricole, dans les bourgades ou les villes du Sud Cameroun (comme Yaoundé, Douala).

Les stratégies des EAF se sont ainsi construites, pour un nombre important d'entre eux, entre trois ou quatre pôles d'activités possibles (cultures, élevage, activités non agricoles, pêche et chasse, voire cueillette). Ces activités ont été développées de façon plus ou moins importante en fonction des possibilités locales et des évolutions du niveau de rémunération du travail et de sécurisation des revenus offerts par les différents secteurs d'activité. Ces stratégies de pluriactivité se sont également construites autour de parcours de vie mêlant des périodes en milieu rural et en milieu urbain.

\section{Des parcours de vie qui mêlent activités agricoles et non agricoles}

La figure 4 montre, pour le site d'Obala, la relation entre la date de naissance de l'individu et le temps de vie qu'il a consacré à l'activité agricole. Pour limiter l'effet de la variation de l'âge des individus sur les résultats obtenus, seul les parcours de vie des 122 individus âgés d'au moins 30 ans au moment de l'enquête (2008), donc nés en 1978 ou avant, ont été analysés. Les 122 individus concernés comprenaient les chefs d'exploitation et leur parentèle.

L'évolution générale mise en évidence par ce graphique était celle d'une diminution au cours du $\mathrm{XX}^{\mathrm{e}}$ siècle du temps de vie consacré à l'activité agricole par les individus. L'indépendance du Cameroun en 1960 a en effet marqué le début d'un important développement des institutions (entre autres, forces armées, appareil judiciaire) et des services publics (santé, enseignement, etc.) de la jeune nation, ainsi que des entreprises privées des secteurs du secondaire et du tertiaire. Les ruraux nés après 1940 ont été

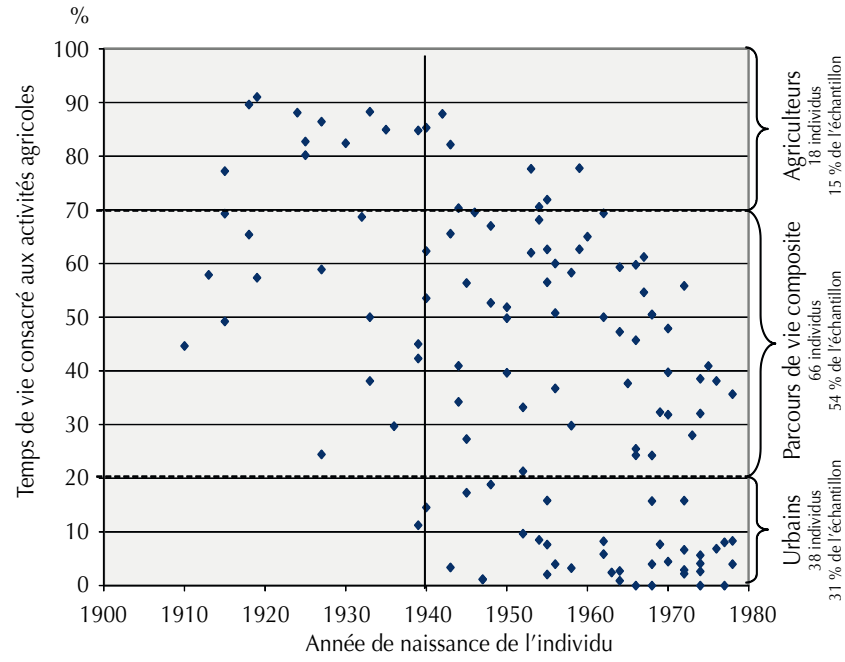

Figure 4 : relation entre la date de naissance et le temps de vie consacré à l'activité agricole.

\section{Tableau III}

Importance relative des revenus du ménage agricole et migration des chefs d'exploitation

Site

Origine des revenus (part en \% du revenu total)

Activités agricoles
Activités non agricoles

Nb. années passées en dehors de l'exploitation par le chef d'exploitation

Culture Elevage Maçonnerie, commerce... Pêche, chasse

\begin{tabular}{|c|c|c|c|c|c|}
\hline Batcham & 51 & 15 & 34 & 0 & 7,8 \\
\hline Galim & 77 & 13 & 10 & 0 & 7,4 \\
\hline Obala & 69 & 6 & 23 & 2 & 10,8 \\
\hline Ayos & 66 & 4 & 16 & 14 & 6,9 \\
\hline
\end{tabular}


fortement attirés par ces nouveaux emplois urbains. Cette diminution du temps de vie consacré à l'activité agricole était essentiellement liée à deux raisons. Premièrement, le temps de vie moyen consacré à la formation scolaire et professionnelle est passé de 2,9 années pour la génération des individus nés entre 1900 et 1940 à 11,2 années pour la génération de ceux nés entre 1941 et 1978 . Deuxièmement, les activités non agricoles ont représenté seulement 18 p. 100 du temps de vie active pour la génération née entre 1900 et 1940 , et en moyenne 41 p. 100 pour la génération née entre 1941 et 1978 .

Parmi les 122 individus enquêtés, 38 (31 p. 100) ont quitté l'agriculture pour construire leur vie majoritairement autour d'activités non agricoles, essentiellement urbaines. Ces individus sont qualifiés d' « urbains » dans le graphique. Ils sont nés dans leur grande majorité après les années 1940 et ont participé à alimenter le mouvement d'exode rural qui s'est développé au Cameroun à partir des années 60 .

Toutefois, ce graphique met surtout en évidence que le phénomène qualifié $\mathrm{d}$ ' « exode rural » (laissant penser que l'on a assisté à un départ définitif de la campagne vers les villes) ne s'est vérifié que partiellement. En effet, de nombreux individus ont développé des parcours professionnels composites, qui ont mêlé activités agricoles et non agricoles, tout au long de leur vie. Dans ces « parcours composites », l'importance des activités agricoles se situait entre celle des individus qualifiés d' « agriculteurs », car ils consacraient la majorité de leur temps de vie aux activités agricoles (> 70 p. 100), et celle des individus qualifiés d' « urbains », pour qui l'activité agricole dans le temps de vie était marginale (<20 p. 100). Ces parcours composites ont concerné 66 des 122 individus enquêtés et donc 54 p. 100 de l'effectif total de l'échantillon. Les individus qualifiés d'agriculteurs étaient au nombre de 18 , soit 15 p. 100 de l'effectif total de l'échantillon. Ces individus aux parcours professionnels composites ont été ainsi dans l'enquête 3,66 fois plus nombreux que ceux qualifiés d'agriculteurs. Ces chiffres ont révélé que l'activité agricole était majoritairement le fait d'individus aux parcours professionnels composites, et non le fait d'agriculteurs qui consacraient l'essentiel de leur vie à cette activité.

Les profils d'évolution obtenus pour les sites de Batcham et d'Ayos ont été similaires à celui d'Obala analysé ici et ont conduit aux mêmes observations. Seul le site de Galim a différé des trois autres (Obala, Batcham et Ayos), puisque l'on a observé dans cette zone maraîchère dynamique une plus faible proportion d'individus ayant développé des parcours composites, et donc une dichotomie plus marquée entre ceux qui vivaient essentiellement des produits de l'agriculture et ceux qui sont partis dans les villes.

\section{DISCUSSION}

L'application à la présente étude des catégories stratégiques proposées par Scoones est d'abord discutée (42), les apports méthodologiques de ce travail sont ensuite analysés.

\section{Diversité des stratégies mobilisées par les acteurs et propositions de la recherche}

Les informations recueillies lors des trois phases de l'étude montrent l'importante diversité des stratégies mises en œuvre par les EAF pour maintenir ou améliorer leurs conditions de vie. Les stratégies d'adaptation identifiées et les enseignements en termes de propositions pour la recherche-développement peuvent être analysés en se référant aux quatre catégories de la grille de classification proposée par Scoones (42). La catégorisation proposée par cet auteur rejoint les observations de la présente étude. Elle a permis de structurer efficacement notre propos et de resituer la place des propositions techniques visant à améliorer les performances techniques dans le cadre plus général des stratégies d'adaptation des EAF.

L'intensification de la production agricole correspond à la première catégorie définie par Scoones (42). Cet objectif d'intensification de la production agricole, qui se traduit généralement par une augmentation des rendements et l'amélioration de la productivité du travail, constitue une priorité forte pour la puissance publique et ses institutions de recherche et de développement agricole. Cette intensification passe, selon ces institutions publiques, en grande partie par la promotion de l'innovation technique. Cependant, cette stratégie d'intensification apparaît peu prioritaire pour les agriculteurs interrogés. Même si certains projets de développement élargissent les objectifs qu'ils fixent au conseil agricole, on observe encore un important décalage entre les voies envisagées par les structures publiques pour améliorer la production agricole et les revenus des agriculteurs et la façon dont les intéressés eux-mêmes s'y prennent pour atteindre ces deux objectifs. Cela explique sans doute en partie les difficultés récurrentes auxquelles se heurtent les projets ou programmes d'intensification agricole au Cameroun et plus largement en Afrique $(30,46)$.

L'extensification de la production agricole constitue la seconde catégorie d'adaptation. Elle correspond à une diminution du niveau des intrants ou de la quantité de main d'œuvre utilisée par hectare de culture. Cette extensification se rencontre sur les cultures pérennes comme le cacao et le café pour lesquelles les exploitants interrogés signalent la diminution de l'usage des intrants comme réponse à la baisse des prix de vente de ces produits d'exportation, en contradiction avec les doses préconisées par la recherche.

La troisième catégorie identifiée par Scoones fait référence à la diversification des moyens d'existence et des activités. La présente étude montre que cette diversification concerne, d'une part, les productions agricoles marchandes et, d'autre part, les types d'activités développées, en particulier les activités non agricoles.

La forte mobilisation par les EAF enquêtés des stratégies de diversification des productions agricoles marchandes incite à développer, en complément des recherches et du conseil agricole organisés par type de culture ou par type d'élevage, des études comparatives et des appuis aux producteurs qui prennent en compte l'ensemble des productions possibles. Il apparaît ainsi utile de développer des analyses technico-économiques et des services de conseil attentifs à l'évolution des nouvelles opportunités qu'offrent les marchés agricoles nationaux et sous-régionaux (comme la Guinée équatoriale, le Gabon). Il s'agit donc d'aller, comme le proposent Faure et coll. (21), vers un conseil qui prenne en compte l'ensemble de l'exploitation agricole et des alternatives productives qui lui sont offertes.

Le développement d'activités et de revenus non agricoles est la stratégie qui est la plus fréquemment citée et mobilisée par les EAF enquêtés. Ceci montre que les représentations décrivant un agriculteur comme un individu qui naît, vit et meurt au sein de son exploitation agricole traduisent de moins en moins la réalité des parcours de vie des individus qui se consacrent aux activités agricoles dans le Grand Sud Cameroun. Le développement des activités non agricoles s'enracine dans la longue période des trois décennies de l'après Indépendance (1960-1990) où la rémunération du travail était plus intéressante en milieu urbain qu'en milieu agricole. Les enquêtes menées par la Société d'études pour le développement économique et social (Sedes) en 1964-65 sur les revenus monétaires annuels moyens des ménages montrent ainsi qu'ils s'élevaient à 55984 FCFA $(\approx 85 € ; 1 € \approx 657$ FCFA $)$ 
dans les zones cacaoyères du Centre Cameroun contre 226380 FCFA $(\approx 344 €)$ pour les ménages demeurant à Yaoundé (43, 44). Ce développement des activités et des revenus non agricoles n'est pas propre au Cameroun (37). Les travaux portant sur ces sujets montrent que 40 p. 100 des revenus des ruraux africains proviennent d'activités non agricoles $(26,39)$. De plus, certaines études indiquent que l'importance de ces revenus d'origine non agricole est en augmentation en Afrique depuis une cinquantaine d'années $(9,27)$. Comme le souligne Brycesson (8), l'abandon durant les décennies 1980-90 des études portant sur l'analyse du monde paysan du point de vue du travail a conduit à une simplification de la réalité. Durant toutes ces années, il a surtout été question « du paysan producteur de denrées agricoles » et une attention insuffisante a été portée à ses capacités de mobilité professionnelle (8). Prendre en compte, au niveau de l'analyse du fonctionnement des exploitations et du conseil, les activités non agricoles des exploitants est alors primordial pour quantifier leur impact sur l'organisation du travail agricole et sur les capacités d'investissement et d'intensification dans l'outil de production agricole $(20,21)$. Il est également important que les analyses macroéconomiques et les politiques publiques agricoles s'intéressent de plus près à la mobilité de la force de travail et des capitaux financiers entre le secteur agricole et les autres secteurs de l'économie nationale.

La quatrième et dernière catégorie identifiée par Scoones (42) se rapporte aux stratégies de migration. Ces stratégies concernent tout d'abord les migrations temporaires vers les zones urbaines qui ont pour objectif le développement d'activités non agricoles, et que l'on peut donc qualifier de "mobilité professionnelle ». Elles concernent également les migrations liées à la recherche de nouvelles terres à mettre en valeur. Les EAF cherchent ainsi à augmenter les surfaces cultivées par actif pour maintenir, voire accroître, leurs revenus monétaires. Cette extension des surfaces cultivées, qui peut dans les zones peu peuplées (Ayos, Galim) se réaliser à l'intérieur du terroir villageois, impose, dans les zones fortement peuplées où les ressources foncières deviennent rares (Batcham et Obala), des «migrations spatiales » temporaires ou définitives. Ces migrations agricoles se développent en particulier sur de vastes fronts pionniers. Ce sont ceux par exemple de la zone de Talba, ville située à $120 \mathrm{~km}$ au nord de Yaoundé, qui portent une part importante de l'expansion cacaoyère actuelle (19), ou celui de Foumbot, ville située à $200 \mathrm{~km}$ au nord-ouest de Yaoundé, qui a largement contribué au formidable accroissement de la production des cultures maraîchères marchandes (47).

$\mathrm{Au}$ Cameroun, la disponibilité en terres est en effet importante, et la mise en valeur de nouvelles surfaces constitue l'un des principaux leviers d'augmentation de la production agricole des exploitants (familiaux, patronaux, capitalistes) et de la nation. L'étude du Programme des Nations unies pour le développement (PNUD) (38) montre, sur la base des deux seuls recensements agricoles disponibles au Cameroun, que la progression de la production agricole est due pour 60 p. 100 à l'augmentation des surfaces mises en valeur, pour 11 p. 100 à l'augmentation de l'intensité culturale (nombre de cycles culturaux pratiqués par campagne) et pour 29 p. 100 à l'augmentation des rendements à l'hectare. L'importance du rôle de l'extension des surfaces dans l'accroissement de la production agricole camerounaise incite à développer des recherches et des politiques de développement agricole moins centrées sur l'amélioration des rendements ou de la productivité du travail et plus attentives à ces dynamiques d'expansion des surfaces cultivées. Les institutions publiques ont en effet un rôle à jouer pour définir avec les partenaires locaux des mesures d'accompagnement permettant que ces dynamiques d'extension agricole offrent une place aux différents types d'exploitants (familiaux, patronaux, capitalistes) et qu'elles concilient au mieux la préservation des ressources naturelles et l'augmentation de la production agricole.

La mobilité spatiale et professionnelle apparait ainsi comme une dimension tout à fait essentielle des stratégies des exploitants. Cette mobilité permet de changer la donne en faisant évoluer les rapports de pouvoir, financiers et symboliques entre groupes sociaux locaux (petits/grands exploitants) et entre les différents membres de la famille (hommes, femmes, jeunes), comme l'a récemment montré Guetat-Bernard pour les femmes de l'Ouest Cameroun (25).

\section{Intérêt et limites de la méthode}

La méthode proposée dans cette étude permet de resituer les propositions techniques de la recherche dans le cadre plus global des stratégies d'adaptation des EAF. Elle repose sur des entretiens semi-directifs, une analyse portée à l'ensemble du système d'activités agricoles et non agricoles, et l'étude des parcours de vie et d'activité sur plusieurs générations.

Cette méthode permet un angle de vue original par rapport aux analyses classiques des facteurs d'adoption des propositions de la recherche centrées sur une liste de critères socio-économiques, techniques ou structurels $(10,41,45)$ sans aisément permettre d'en analyser la cohérence par rapport à des objectifs de production. Elle permet d'aborder la complexité et la richesse des stratégies paysannes et d'en comprendre les évolutions et les déterminants. L'analyse des stratégies des EAF nécessite d'expliciter non seulement les décisions prises au niveau de leurs systèmes de production et de leurs pratiques techniques, mais également de préciser leurs choix au niveau de leurs systèmes d'activités agricoles et non agricoles. En outre, la prise en compte de la pluriactivité des EAF doit s'établir non seulement à un temps « $t$ » mais aussi dans le cadre des parcours professionnels sur l'ensemble de la vie de l'exploitant pour identifier des changements de stratégies en lien avec la variation du contexte socio-économique. Cela nécessite alors de construire des bases de données décrivant les parcours professionnels et les différentes sources de revenus monétaires des différents membres de l'exploitation agricole pour analyser ces stratégies sur des pas de temps longs.

Cette démarche complète de façon utile les méthodes proposées pour l'analyse des trajectoires de vie qui insistent surtout sur l'étude des bifurcations et de leurs causes $(5,24)$. Utilisée ici dans le cadre de systèmes de production dominés par les cultures annuelles et pérennes, elle peut également être mobilisée pour l'analyse des stratégies des éleveurs ou des agropasteurs pour qui la mobilité spatiale, l'intégration entre systèmes de production et le temps long $(12,15)$ sont aussi des dimensions importantes.

\section{- CONCLUSION}

La démarche d'investigation et les méthodes mobilisées ont permis de vérifier l'hypothèse de départ : l'intensification via l'adoption de propositions techniques ne représente qu'une des stratégies développées par les EAF pour maintenir ou améliorer leurs conditions de vie. Le développement des activités non agricoles, l'extension des surfaces cultivées, la diversification des productions agricoles marchandes apparaissent comme des stratégies d'adaptation bien plus fréquemment utilisées par les EAF que l'amélioration des performances des itinéraires techniques par l'adoption de propositions techniques. Les présents travaux montrent en particulier que les mobilités spatiales et professionnelles sont au cœur de leurs stratégies. 
Cette étude souligne ainsi le fait que l'amélioration des itinéraires techniques et des performances des exploitations familiales ne saurait constituer la seule orientation des politiques de recherche et de développement du secteur agricole. L'augmentation des mobilités spatiales et professionnelles des exploitants agricoles encourage à développer des politiques de recherche et de développement plus attentives à la gestion des territoires et des hommes et aux interactions entre les différents secteurs de l'économie nationale.

\section{BIBLIOGRAPHIE}

1. ALARY V., COURADE G., JANIN P., 1994. Permanence et flexibilité des cacaoculteurs béti à l'heure des ajustements. In : Courade G., coord., Le village camerounais à I'heure de l'ajustement. Paris, France, Karthala, p. 170-183. (Coll. Economie et développement)

2. BANQUE MONDIALE, 2008. Rapport sur le développement dans le monde 2008. L'agriculture au service du développement, vers. abrégée. Washington, DC, USA, World Bank, $34 \mathrm{p}$.

3. BARBIER J.C., COURADE G., GUBRY P., 1977. L'exode rural au Cameroun. Yaoundé, Cameroun, Office national de la recherche scientifique et technique, $113 \mathrm{p}$.

4. BARDIN L., 1993. L'analyse de contenu. Paris, France, PUF, 291 p.

5. BIDART C., 2006. Crise, décisions et temporalités : autour des bifurcations biographiques, trajectoires sociales et bifurcations. Cah. int. Soc., $120: 29-57$

6. BROOKS N., 2003. Vulnerability, risk and adaptation : a conceptual framework. Norwich, UK, Tyndall Centre for Climate Change Research, University of East Anglia, 19 p.

7. BROSSIER J., CHIA E., MARSHALL E., PETIT M., 1997. Gestion de l'exploitation agricole familiale. Eléments théoriques et méthodologiques. Dijon, France, Educagri, 217 p.

8. BRYCESSON D.F., 1999. African rural labour, income diversification and livelihood approaches: a long term development perspective. ASC Working Paper 35/1999. Leiden, Netherlands, Afrika-Studiecentrum, $40 \mathrm{p}$.

9. BRYCESSON D.F., JAMAL V., 1997. Farewell to farms: deagrarianisation and employment in Africa. Aldershot, UK, Ashgate, $265 \mathrm{p}$.

10. BYRNE F., ROBERTSON M.J., BATHGATE A., HOQUE Z., 2010. Factors influencing potential scale of adoption of a perennial pasture in a mixed crop-livestock farming system. Agric. Syst., 103: 453-462.

11. CharaudeaU P., MaINGUENEAU D., 2002. Dictionnaire d'analyse du discours. Paris, France, Seuil, $662 \mathrm{p}$.

12. CIALDELLA N., DOBREMEZ L., MADELRIEUX S., 2009. Livestock farming systems in urban mountain regions: differentiated paths to remain in time. Outlook Agric., 38: 127-135.

13. COURADE G., 1994. Le village camerounais à l'heure de la crise. Paris, Karthala, 410 p. (Coll. Economie et développement)

14. DANNEFER D., 2003. Toward a global geography of the life course. Challenges of late modernity for life course theory. In: Mortimer J.T. Shanahan M.J. Eds, Handbook of the life course. New York, USA, p. 647659.

15. DEDIEU B., INGRAND S., 2010. Incertitude et adaptation : cadres théoriques et application à l'analyse de la dynamique des systèmes d'élevage. Prod. Anim., $23: 81-90$.

16. DIRECTION DE L'AGRICULTURE, 1969. La politique agricole du Cameroun oriental, dans la perspective du III plan quinquennal. Yaoundé, Cameroun, secrétariat d'Etat au Développement rural, direction de l'Agriculture, $30 \mathrm{p}$.

17. DJAMEN NANA P., DJONNEWA A., HAVARD M., LEGILE A., 2003. Former et conseiller les agriculteurs du Nord-Cameroun pour renforcer leurs capacités de prise de décision. Cah. Agric., 12 : 241-245.

18. DONGMO J.L., 1981. Le dynamisme Bamiléké (Cameroun). In : La maîtrise de l'espace agraire, vol. 1. Yaoundé, Cameroun, Université de Yaoundé, $422 \mathrm{p}$.

19. ELONG J.G., 2004. Eton et Manguissa, de la Lékié au Mbam-et-Kim : jeux et enjeux fonciers (Centre-Cameroun). Cah. Outre-Mer Afr. (226227) : $1-15$
20. FAURE G., DUGUE P., BEAUVAL V., 2004. Conseil à l'exploitation familiale. Expériences en Afrique de l'Ouest et du Centre. Guide Pratique. Paris, Gret / Montpellier, Cirad (coll. Agridoc), 127 p.

21. FAURE G., DUGUE P., BEAUVAL V., 2007. Conseil aux exploitations familiales. In : Gafsi M., Dugué P., Jamin J.-Y., Brossier J., Eds., Exploitations agricoles familiales en Afrique de l'Ouest et du Centre : enjeux, caractéristiques et éléments de gestion. Paris, France, Quae, p. 369-402.

22. FOLKE C., COLDING J., BERKES F., 2002. Building resilience for adaptive capacity in social-ecological systems. In: Berkes F., Folke C., Eds, Navigating social-ecological systems: building resilience for complexity and change. Cambridge, UK, Cambridge University Press.

23. FRANQUEVILLE A., 1987. Une Afrique entre le village et la ville. Les migrations dans le sud du Cameroun. Paris, France, Orstom, 646 p.

24. GROSSETTI M., 2006. L'imprévisibilité dans les parcours sociaux. Trajectoires sociales et bifurcations. Cah. int. Soc., 120 : 5-28.

25. GUETAT-BERNARD H., 2011. Développement rural et rapport de genre. Mobilité et argent au Cameroun. Rennes, France, Presses universitaires de Rennes, 213 p.

26. HAGGBLADE S., HAZELL P., BROWN J., 1989. Farm-nonfarm linkages in rural sub-Saharan Africa. World Dev., 17: 1173-1201.

27. HAGGBLADE S., HAZELL P., REARDON T., 2010. The rural non-farm economy, prospects for growth and poverty reduction. World Dev., 38: 1414-1441.

28. LEPLAIDEUR A., 1987. Stratégies paysannes autour des systèmes cacaoyers et vivriers en zone Centre-Sud forestière camerounaise. In : Actes du colloque Cirad/Mesru, Etats, développement, paysans, sept. 1985, p. 74-85.

29. LOSCH B., FUSILLIER J.L., DUPRAZ P., 1991. Stratégies des producteurs en zone caféière et cacaoyère du Cameroun. Quelles adaptations à la crise? Montpellier, France, Cirad, 252 p. (Coll. Systèmes agraires, 12)

30. MEINKE H., BAETHGEN W.E., CARBERRY P.S., DONATELLI M., HAMMER G.L., SELVARUJU R., STOCKLE C.O., 2001. Increasing profits and reducing risks in crop production using participatory systems simulation approaches. Agric. Syst., 70: 493-513.

31. MIKOLASEK O., BARLET B., CHIA E., POUOMOGNE V., TOMEDI E.T.M., 2009. Développement de la petite pisciculture marchande au Cameroun : la recherche-action en partenariat. Cah. Agric., 18 : 270-276. 32. MINADER-MINEPIA, 2007. Document de présentation du programme Amélioration de la compétitivité des exploitations familiales agropastorales. Yaoundé, Cameroun, Minader-Minepia, 102 p.

33. MINEPAT/CTSE, 2009. Document de stratégies pour la croissance et l'emploi. Cadre de référence de l'action gouvernementale pour la période 2010-2019. Yaoundé, Cameroun, Minepat, 104 p.

34. MINISTERE DE L'AGRICULTURE, 1981. Ve Plan quinquennal. Secteur agricole. Yaoundé, Cameroun, ministère de l'Agriculture, $157 \mathrm{p}$.

35. MINISTERE DE L'AGRICULTURE, 2002. Stratégie de développement du secteur rural. Yaoundé, Cameroun, ministère de l'Agriculture, $34 \mathrm{p}$.

36. MINPAT, 1999. Etudes socio-économiques régionales au Cameroun. Cadrage national. Yaoundé, Cameroun, Minpat / PNUD-OPS, 423 p.

37. PAUL J.-L., BORY A., BELLANDE A, GARGANTA E., FABRI A., 1994. Quel système de référence pour la prise en compte de la rationalité de I'agriculteur : du système de production agricole au système d'activité. Cah. Rech. Dév., 39 : 7-19.

38. PNUD-FAO, 1990. Bilan diagnostic et perspectives de la colonisation des terres au Cameroun. Yaoundé, Cameroun, ministère de l'Agriculture, division des projets agricoles, $117 \mathrm{p}$.

39. REARDON T., 1997. Using evidence of household income diversification to inform study of the rural nonfarm labor market in Africa. World Dev., 25: 735-747.

40. SANTOIR C., 1992. Sous I'empire du cacao. Etude diachronique de deux terroirs camerounais. Paris, France, Orstom, p. 191. (Coll. A travers champs)

41. SATTLER C., NAGEL U.J., 2010. Factors affecting farmers' acceptance of conservation measures. A case study from north-eastern Germany. Land Use Policy, 27: 70-77.

42. SCOONES I.,1998. Sustainable rural livelihoods: a framework for analysis. Brighton, UK, IDS, p. 72. 
43. SEDES, 1966. Enquête sur le niveau de vie à Yaoundé, 1964-1965. Paris, France, Société d'études pour le développement économique et social, p. 247

44. SEDES, 1966. Le niveau de vie des populations de la zone cacaoyère du Centre Cameroun. Résultats définitifs de l'enquête sur la nutrition et les budgets des ménages ruraux exécutée dans la zone en 1964-1965. Paris, France, Société d'études pour le développement économique et social, p. 117-182

45. SOMDA J., NIANOGO A.J., NASSA S., SANOU S., 2002. Soil fertility management and socio-economic factors in crop-livestock systems in Burkina Faso: a case study of composting technology. Ecol. Econ., 43: 175-183.

46. VAN RIJN F., BULTE E., ADEKUNLE A., 2012. Social capital and agricultural innovation in sub-Saharan Africa. Agric. Syst., 108: 112-122.
47. VOLLANT S., 2003. La frontière agraire du sud de Foumbot : mobilités et modification du système de production. Maîtrise Géogr., Université de Toulouse Le Mirail, Toulouse, France, 245 p.

48. WEBER J., 1979. Logiques paysannes et rationalité technique : illustrations camerounaises. In : Actes colloque Maîtrise de I'espace agraire et développement en Afrique tropicale, logique paysanne et rationalité technique, Ouagadougou, Burkina Faso, 4-8 déc. 1978. Paris, France, Orstom, p. 311-315. (Coll. Mémoires, n 89)

49. YUNG J.M., ZASLAVSKY J., 1992. Pour une prise en compte des stratégies des producteurs. Montpellier, France, Cirad, 74 p.

Mis en ligne en juin 2013

\section{Summary}

Pédelahore P., Tchatchoua R., Tonka M., Ntsama M., Andrieu $\mathbf{N}$. Place of the adoption of technical proposals from research in farmers' adaptation strategies

Technical innovation is often presented as the main lever to improve economical performances and livelihoods of family farmers. It is thus a way to face the variability of the socio-economical environment. The aim of this study was to analyze the role of the adoption of technical proposals from research within the range of the adaptation strategies used by farmers to maintain or even improve their livelihoods and that of their descendants. Semi-structured and structured interviews carried out on a sample of representative family farmers of South Cameroon showed that migrations, off farm activities, increase of cultivated areas, and cash crop diversification were adaptation strategies more often used by farmers than the adoption of technical proposals. The study highlighted that improving crop management sequences and performances of family farming systems could not be the sole orientation of research and development policies. The increase of farmers spatial and professional mobility points the need to develop research and development policies that focus more closely on territorial and farmers' management and on interactions between the different sectors of the national economy.

Keywords: Family farm - Innovation adoption - Development policy - Cameroon.

\section{Resumen}

Pédelahore P., Tchatchoua R., Tonka M., Ntsama M., Andrieu N. Restituir la adopción de propuestas técnicas de la investigación dentro de las estrategias de adaptación de los productores agrícolas familiares

La innovación técnica se presenta a menudo como el principal incentivo de mejoramiento de los rendimientos económicos y de las condiciones de vida de los productores agrícolas familiares (PAF). Constituye por ende un medio de adaptación frente a la variabilidad del medio socio económico. El objetivo de este estudio fue el de analizar el papel del recurso de la adopción de propuestas técnicas derivadas de la investigación en el seno del conjunto de estrategias de adaptación movilizadas por los productores para preservar o mejorar sus condiciones de vida y las de sus descendientes. Mediante de la realización de entrevistas semi directivas y directivas, en una muestra representativa de PAF en el Gran Sur de Camerún, este estudio demostró que el desarrollo de las migraciones y de las actividades no agrícolas, la extensión de superficies cultivadas y la diversificación de producciones agrícolas mercantiles fueron estrategias de adaptación más frecuentemente movilizadas que la adopción de propuestas técnicas. Este estudio realza el hecho de que el mejoramiento de los itinerarios técnicos y de los rendimientos de las explotaciones familiares no puede constituir la única orientación de las políticas de investigación y de desarrollo. El aumento de las movilizaciones espaciales y profesionales de los productores fomenta el desarrollo de políticas de investigación y de desarrollo más atentas a la gestión de los territorios y de los hombres y a las interacciones entre los diferentes sectores de la economía nacional.

Palabras clave: Explotación agrícola familiar - Adopción de innovación - Política de desarrollo - Camerún. 
